\title{
Guidelines for the labelling of leucocytes with ${ }^{99 m}$ Tc-HMPAO
}

\author{
Erik F. J. de Vries • Manel Roca • Francois Jamar • \\ Ora Israel • Alberto Signore
}

Published online: 3 March 2010

(C) The Author(s) 2010. This article is published with open access at Springerlink.com

\begin{abstract}
We describe here a protocol for labelling autologous white blood cells with ${ }^{99 \mathrm{~m}} \mathrm{Tc}-\mathrm{HMPAO}$ based on previously published consensus papers and guidelines. This protocol includes quality control and safety procedures and is in accordance with current European Union regulations and International Atomic Energy Agency recommendations.
\end{abstract}

Keywords Guidelines - White blood cells .

Scintigraphy $\cdot{ }^{99 \mathrm{~m}} \mathrm{Tc}-\mathrm{HMPAO} \cdot$ Infection

$\overline{\text { Erik F. J. de Vries and Manel Roca contributed equally to this work. }}$

\section{E. F. J. de Vries}

Department of Nuclear Medicine and Molecular Imaging, University Medical Center Groningen, University of Groningen, Groningen, The Netherlands

M. Roca

Radiopharmacy Unit, Nuclear Medicine Department,

Hospital Universitario de Bellvitge,

Barcelona, Spain

F. Jamar

Nuclear Medicine, Université Catholique de Louvain,

Brussels, Belgium

O. Israel

Departments of Nuclear Medicine, Rambam Health Care Campus, Haifa, Israel

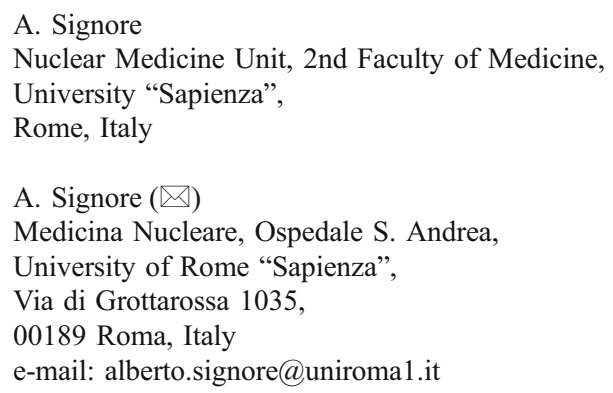

\section{Purpose}

The aim of this document is to provide information on the preparation and quality control of white blood cells (WBC) labelled with ${ }^{99 \mathrm{~m}} \mathrm{Tc}$-exametazime $\left({ }^{99 \mathrm{~m}} \mathrm{Tc}\right.$-hexamethylpropyleneamine oxime; $\left.{ }^{99 \mathrm{~m}} \mathrm{Tc}-\mathrm{HMPAO}\right)$ that can assist nuclear medicine practitioners, radiopharmacists, technicians and other individuals involved. The corresponding guidelines of the Society of Nuclear Medicine, the consensus protocol of the International Society of Radiolabelled Blood Elements (ISORBE), recommendations of the International Atomic Energy Agency, several national guidelines, and the most relevant literature were taken into consideration and were partially integrated into this text [1-21]. The present guidelines, therefore neither arise from a consensus conference nor from an evidence-based meta-analysis, but were produced by a panel of experts based on data from peerreviewed literature, as well as their own experience and knowledge of colleagues from all over the world that was shared at meetings, at congresses and during collaborative scientific work including multicentre studies.

Different European countries have different recommendations and laws regarding the specific matter of production of extemporaneous radiopharmaceuticals, in particular when labelled autologous cells are considered, since they cannot be sterilized after synthesis. We performed a survey in Europe, to which the national delegates of 14 countries officially replied and four others contributed verbally. The results of this survey are summarized in Table 1 and demonstrate overall that WBC labelling is a well-established technique in Europe, and is mainly performed by trained personnel under sterile conditions in a laminar flow cabinet (class A). However, the requirements for the environment in which the flow cabinet is located vary greatly among countries. Thus, different rules apply among the European countries. 
Table 1 Results of a survey about WBC labelling amongst the national delegates of the EANM

\begin{tabular}{|c|c|c|c|}
\hline $\begin{array}{l}\text { How many centres perform } \\
\text { WBC labelling in your } \\
\text { country? }\end{array}$ & $\begin{array}{l}\text { Are there national rules that } \\
\text { prescribe how WBC should } \\
\text { be labelled? }\end{array}$ & $\begin{array}{l}\text { According to national rules, } \\
\text { what personnel are allowed } \\
\text { to label WBC? }\end{array}$ & $\begin{array}{l}\text { According to national rules, what } \\
\text { kind of environment is required } \\
\text { for labelling WBC? }\end{array}$ \\
\hline Range $0-66.7 \%$ & By law, 5\% (1) & Nuclear physicians, $5 \%$ (1) & Class-A hood, $22 \%$ (4) \\
\hline Mean $32.6 \%$ & Recommendations, $67 \%$ (12) & Radiopharmacists, $16 \%$ (3) & Class A hood in B, $28 \%(5)$ \\
\hline SD $23.9 \%$ & Conform to cGRPP, $17 \%$ (3) & Pharmacists, 11\% (2) & Class A hood in C, $16 \%$ \\
\hline \multirow[t]{2}{*}{ Median $41.4 \%$} & No rules, $27 \%(5)$ & Trained personnel, 55\% (10) & Class A hood in D, $16 \%$ \\
\hline & & No rules, $16 \%$ (3) & No rules, $11 \%(2)$ \\
\hline
\end{tabular}

cGRPP current good radiopharmacy practice.

This protocol aims to guide labelling of WBC in accordance with currently effective European Union regulations. In addition, the specific rules in various countries must be complied with. The procedure may therefore have to be adjusted to local rules, needs and the equipment available.

\section{Background information}

Scintigraphy with labelled autologous WBCs is a widely used method to detect sites of infection. In the mid 1970s, ${ }^{111}$ In-oxine was introduced as a nonselective labelling agent for WBC scintigraphy. Although ${ }^{111}$ In-oxine-labelled WBC have been successfully used in the field of infection/ inflammation scintigraphy, over the years the labelling agent has been largely replaced by ${ }^{99 \mathrm{~m}} \mathrm{Tc}-\mathrm{HMPAO}$, because of the favourable physical characteristics, availability, cost and lower radiation burden (Table 2) of ${ }^{99 \mathrm{~m}} \mathrm{Tc}$ as compared with ${ }^{111} \mathrm{In}$. Since ${ }^{111}$ In-oxine-labelled WBC are still in use in several centres and for specific clinical indications, the labelling procedure of WBC with this radiopharmaceutical are covered in separate guidelines.

${ }^{99 \mathrm{~m}}$ Tc-HMPAO kit preparations have been commercially available since 1988. Upon reconstitution of the HMPAO kit (containing both D-and L-isomers) with ${ }^{99 \mathrm{~m}} \mathrm{Tc}$-pertechnetate from a fresh generator eluate (preferably within $30 \mathrm{~min}$ of elution) a lipophilic complex is formed. The lipophilic complex is transformed into free ${ }^{99 \mathrm{~m}} \mathrm{Tc}-$ pertechnetate and a hydrophilic ${ }^{99 \mathrm{~m}} \mathrm{Tc}-\mathrm{HMPAO}$ complex in aqueous solution over time. Only freshly prepared ${ }^{99 \mathrm{~m}} \mathrm{Tc}-\mathrm{HMPAO}$ should therefore be used for leucocyte labelling (within $20 \mathrm{~min}$ of preparation) since only the lipophilic ${ }^{99 m} \mathrm{Tc}-\mathrm{HMPAO}$ complex can freely cross the cell membrane of WBC and is subsequently trapped inside the cell.

Two mechanisms have been suggested to be responsible for the retention of ${ }^{99 \mathrm{~m}} \mathrm{Tc}-\mathrm{HMPAO}$ inside the cell: (1) conversion of the lipophilic ${ }^{99 m} \mathrm{Tc}-\mathrm{HMPAO}$ complex into a hydrophilic complex by reducing agents such as glutathione, and (2) binding of ${ }^{99 \mathrm{~m}} \mathrm{Tc}-\mathrm{HMPAO}$ to nondiffusible proteins and cell organelles. Some release of ${ }^{99 \mathrm{~m}} \mathrm{Tc}-\mathrm{HMPAO}$ from the labelled $\mathrm{WBC}$ after reinjection into the patient is observed, resulting in undesired accumulation of radioactivity in the gastrointestinal and urinary tracts. For WBC scintigraphy, either mixed leucocytes or isolated granulocytes can be used. When mixed leucocytes are labelled with ${ }^{99 \mathrm{~m}} \mathrm{Tc}$-HMPAO, about $70-80 \%$ of the radioactivity is bound to granulocytes. Labelled mixed leucocytes can display higher blood pool activity than labelled isolated granulocytes, especially in early images, due to the presence of labelled lymphocytes and residual erythrocytes.

\section{Common indications for ${ }^{99 \mathrm{~m}}$ Tc-HMPAO WBC scintigraphy}

${ }^{99 \mathrm{~m}} \mathrm{Tc}-\mathrm{HMPAO}$-labelled WBC scintigraphy may be used to detect and localize any occult site of infection and to

Table 2 Radiation dosimetry for ${ }^{99 \mathrm{~m}} \mathrm{Tc}-\mathrm{HMPAO}$ labelled leucocytes

\begin{tabular}{llll}
\hline Population & Administered activity $(\mathrm{MBq})$ & $\begin{array}{l}\text { Organ receiving the largest radiation } \\
\text { dose (spleen) }(\mathrm{mGy} / \mathrm{MBq})\end{array}$ & $\begin{array}{l}\text { Effective dose equivalent } \\
(\mathrm{mSv} / \mathrm{MBq})\end{array}$ \\
\hline Adults & $185-370$ & 0.15 & 0.011 \\
Children (5 years old) & $3.7-7.4 / \mathrm{kg}$ & 0.48 & 0.034
\end{tabular}

International Commission on Radiological Protection. Annals of the ICRP, Publication 80, Radiation Dose to Patients from Radiopharmaceuticals, Ed. J Valentin, Elsevier Science, Oxford, UK; 1999, p. 67. 
determine the extent of the process in various disorders, including:

- Osteomyelitis of the appendicular skeleton

- Infected joint and vascular prosthesis

- Diabetic foot

- Fever of unknown origin

- Postoperative abscesses

- Lung infections

- Endocarditis

- Inflammatory bowel disease

- Neurological infections

- Infected central venous catheters or other devices

Inflammatory bowel disease and kidney infections are better imaged with ${ }^{111}$ In-oxine-labelled WBC, because of the lower release of the radiopharmaceutical and its metabolites from labelled cells, resulting in lower nonspecific gastrointestinal and urinary tract activity.

\section{Precautions}

During the labelling procedure, blood and blood components from the patient, who could potentially be infected with pathogens, need to be handled. To prevent contamination of the operator who is performing the labelling, waterproof gloves should be worn throughout the procedure. Special caution should be taken when handling needles.

Since ${ }^{99 \mathrm{~m}}$ Tc-HMPAO-labelled WBC have to be reinjected into the patient, strict aseptic conditions are required for the labelling procedure. For this purpose, only sterile reagents and disposable plastic-ware should be used, and sterile gloves, cap and mask should be worn. Usually, the labelling of WBC is performed in a laminar flow cabinet or cell isolator, installed according to local regulations. Recently, certified sterile closed-kit labelling devices have become available that may represent a good approach to WBC labelling with further protection for patient and operator, despite the fact that they have to be used in a sterile cabinet as well.

Simultaneous labelling of WBC from multiple patients is discouraged in order to prevent possible cross-contamination. Labelling of WBC from different patients should be carried out at physically separated locations unless closed devices are used. At all times correct identification of the patient's blood products should be guaranteed. All syringes, tubes and any material in contact with the patient's blood components should be clearly labelled with the patient's name, bar-code and/or colour code.

During the labelling of WBC with ${ }^{99 \mathrm{~m}} \mathrm{Tc}-\mathrm{HMPAO}$ care should be taken that leucocytes are not damaged, as this would result in leakage of the radioactivity from the cells, adhesion of labelled leucocytes to the vascular endothelium (especially in the microvasculature of the lungs) and loss of motility. To avoid degradation of the radiopharmaceutical and radiation damage to labelled cells, ${ }^{99 \mathrm{~m}} \mathrm{Tc}-\mathrm{HMPAO}$ labelled WBC should be reinjected as soon as possible, but not later than $1 \mathrm{~h}$ after labelling.

Labelling of mixed leucocytes causes radiation damage to the lymphocytes as a result of self-irradiation by low-energy Auger electrons. However, since the lymphocytes are unable to divide after labelling and are eliminated through apoptosis and phagocytosis, the risk of lymphoid malignancies after administration of ${ }^{99 \mathrm{~m}} \mathrm{Tc}-\mathrm{HMPAO}$-labelled mixed leucocytes is considered to be negligible.

\section{Procedure}

\section{A. Isolation of WBCs}

A1. Collection of blood Fill a 60-ml syringe with $9 \mathrm{ml}$ of acidcitrate-dextrose anticoagulant solution $(\mathrm{ACD}$; formulation A according to the European Pharmacopoeia, consisting of $0.73 \mathrm{~g}$ of anhydrous citric acid, $2.2 \mathrm{~g}$ of sodium citrate dihydrate and $2.45 \mathrm{~g}$ of dextrose monohydrate in $100 \mathrm{ml}$ of water for injection) and add $51 \mathrm{ml}$ of the patient's blood to this syringe. Use a needle with an inner diameter of at least $20 \mathrm{G}$ to prevent damage to the WBC. Damage is related to shear stress due to high laminar flow in small needles when using large syringes. Blood withdrawal should be slow and smooth in order to prevent the formation of bubbles and foaming. Mix the bloodACD solution by gently turning the syringe end over end a few times (do not mix by shaking). At least, $2 \times 10^{8}$ leucocytes are required to achieve a good labelling efficiency (LE). In patients with neutropenia $\left(<2 \times 10^{3}\right.$ neutrophils $\left./ \mathrm{mm}^{3}\right)$, an additional syringe of ACD anticoagulant and blood may be needed. Although the use of smaller volumes of blood (down to as little as $20 \mathrm{ml}$ ) has been described in the literature, this is not recommended, because it tends to reduce the LE.

Smaller blood volumes can be drawn from children, depending on feasibility and considering that the activity is determined according to body weight (see EANM guidelines Paediatric Card 2008; https://www.eanm.org/scientific_info/ dosagecard/). In this case, the use of smaller syringes and needles is advised (use multiple 10-ml syringes containing $1.8 \mathrm{ml}$ of ACD).

A2. Isolation of cell-free plasma Dispense $15 \mathrm{ml}$ of the blood-ACD solution into a Falcon centrifugation tube and centrifuge at $2,000 \mathrm{~g}$ at room temperature for $10 \mathrm{~min}$. Separate the cell-free plasma (CFP) from the pellet. The CFP will be used as the medium for labelling and cell resuspension. Isotonic phosphate-buffered saline, $\mathrm{pH} 7.4$ 
(PBS), or a $0.9 \%$ aqueous solution of sodium chloride (saline) could be used as an alternative to CFP, although CFP is a more physiological medium. There is no scientific evidence that supports preference of one medium over another.

A3. Isolation of mixed leucocytes First, erythrocytes are allowed to sediment with the aid of 2-hydroxyethyl starch (10\% HES, pharmaceutical grade). While several formulations of HES plasma expander are commercially available, it is highly recommended to use high molecular weight HES $200 / 0.5$ or $200 / 0.6$ (mean molecular weight of 200 $\mathrm{kDa}$ ), since formulations of lower molecular weight HES do not work as well as sedimentation agents for getting leucocyte-rich plasma (LRP) from blood. Add $4.5 \mathrm{ml}$ of $10 \%$ HES to the remaining $45 \mathrm{ml}$ of the blood-ACD mixture. Although a ratio of blood-ACD mixture to HES of 10:1 is recommended, the concentration of HES can be increased up to a ratio of $5: 1$ for patients with polycythaemia or sickle-cell anaemia. Gently turn the syringe end over end a few times. Place the syringe with the opening up and allow the erythrocytes to sediment. This takes 30 to $45 \mathrm{~min}$. As an alternative method to separate erythrocytes from LRP, centrifugation at low speed has been described [20], but this method has not been widely applied yet. The centrifugation method is faster than the sedimentation method, but gives rise to more erythrocyte contamination.

Collect the LRP in a Falcon centrifuge tube. This can be achieved via a long lumbar needle or a butterfly needle of at least $20 \mathrm{G}$ by gently pushing the piston of the syringe up, without disturbing the erythrocytes. An alternative way to transfer the LRP is to use a 5-inch Kwill filling tube. Centrifuge the LRP at $150 \mathrm{~g}$ for $5 \mathrm{~min}$. Application of higher centrifugal forces should be avoided, since this would lead to increased platelet contamination. Lower centrifugal forces can be applied if the centrifugation time is adjusted accordingly. (NB: To avoid resuspension of the leucocytes, the centrifuge's brakes should be switched off in all centrifugation steps.)

Remove the platelet-rich plasma (PRP) and gently resuspend the mixed leucocyte pellet. As an optional step, the pellet can be washed with CFP (from step A2; or alternatively PBS or saline) to reduce the number of contaminating platelets. For this purpose, gently resuspend the pellet in $3 \mathrm{ml}$ of CFP (or alternatively PBS or saline), centrifuge at $150 \mathrm{~g}$ for $5 \mathrm{~min}$ and remove the supernatant from the leucocyte pellet. Gently resuspend the mixed leucocytes in $1 \mathrm{ml}$ of fresh CFP (or alternatively PBS or saline) and use this cell suspension for labelling (section B).

A4. Isolation of granulocytes (optional) Although labelling of mixed leucocytes (from A3) is recommended, purified granulocytes can be used instead. When desired, granulo- cytes can be isolated from mixed leucocytes by gradient centrifugation. For this purpose, prepare gradient solution A and gradient solution B. Gradient solution A consists of $1.0 \mathrm{ml}$ of $9 \% \mathrm{NaCl}, 5.5 \mathrm{ml}$ Percoll (colloidal silica particles coated with polyvinylpyrrolidone; GE Healthcare, Little Chalfont, UK, or Sigma-Aldrich, St Louis, MO) and $3.4 \mathrm{ml}$ water for injection, and gradient solution B consists of $1.0 \mathrm{ml}$ of $9 \% \mathrm{NaCl}, 7.0 \mathrm{ml}$ Percoll and $1.9 \mathrm{ml}$ water for injection. Carefully inject with a syringe $4 \mathrm{ml}$ of gradient solution A and subsequently $4 \mathrm{ml}$ of gradient solution B under the mixed leucocyte suspension (i.e. at the bottom of the tube). Take care that the cell suspension and the gradient solutions do not mix. Centrifuge the gradient at $150 \mathrm{~g}$ for $30 \mathrm{~min}$. The granulocytes are now visible as a white layer between the gradient solutions, whereas the mononuclear cells and residual platelets are on the top of the gradient and erythrocytes are in the pellet. Carefully aspirate the granulocyte layer with a plastic Pasteur-type pipette and dispense them into a Falcon tube. Add $10 \mathrm{ml}$ of saline solution (or PBS) and centrifuge at $150 \mathrm{~g}$ for $10 \mathrm{~min}$. Remove the supernatant and gently resuspend the granulocyte pellet in $1 \mathrm{ml}$ of CFP (or PBS or saline). As an easy alternative to Percoll, Lymphoprep has been used [22], but it has not been licensed for patient use, and therefore ethical approval is required.

\section{B. Labelling of WBCs with ${ }^{99 \mathrm{~m}} \mathrm{Tc}-\mathrm{HMPAO}$}

Prepare ${ }^{99 \mathrm{~m}} \mathrm{Tc}-\mathrm{HMPAO}$ using freshly eluted ${ }^{99 \mathrm{~m}} \mathrm{Tc}$-pertechnetate. Do not use a stabilizing agent (methylene blue, cobalt) or PBS in the preparation of ${ }^{99 \mathrm{~m}} \mathrm{Tc}-\mathrm{HMPAO}$. Because ${ }^{99 \mathrm{~m}} \mathrm{Tc}-\mathrm{HMPAO}$ is unstable in aqueous solutions, the radiopharmaceutical should be prepared immediately before use. Periodically, perform quality control of the ${ }^{99 \mathrm{~m}} \mathrm{Tc}-\mathrm{HMPAO}$ preparation according to the procedure described by the manufacturer in the accompanying leaflet. Add $1 \mathrm{ml}$ of freshly prepared ${ }^{99 \mathrm{~m}} \mathrm{Tc}-\mathrm{HMPAO}$ (approximately $750-1,000 \mathrm{MBq}$ ) in saline solution to the mixed leucocyte cell suspension (or purified granulocytes) and incubate for $10 \mathrm{~min}$ at room temperature. Although the package insert of HMPAO (Ceretec; GE Healthcare, Little Chalfont, UK) indicates that ${ }^{99 \mathrm{~m}} \mathrm{Tc}-\mathrm{HMPAO}$ should be prepared in $5 \mathrm{ml}$ and that the whole dose should be used for a single labelling, there is enough scientific evidence to support the use of smaller volumes of ${ }^{99 \mathrm{~m}} \mathrm{Tc}-\mathrm{HMPAO}$ that result in higher LE. During incubation, gently swirl the cell suspension periodically to prevent sedimentation of the cells. After the incubation is complete, add at least $3 \mathrm{ml}$ (preferably up to $10 \mathrm{ml}$ ) of CFP, and centrifuge at $150 \mathrm{~g}$ for 5 min. If necessary, additional CFP can be obtained by centrifugation of the PRP (step A3) at 2,000g for $10 \mathrm{~min}$. Alternatively, PBS or saline could be used instead of CFP. After centrifugation, remove the supernatant containing 
unbound ${ }^{99 \mathrm{~m}} \mathrm{Tc}-\mathrm{HMPAO}$ and measure the amount of radioactivity in the pellet and in the supernatant to calculate the LE. Gently resuspend the pellet containing the labelled mixed leucocytes in 3-5 $\mathrm{ml}$ of CFP. Dispense the patient dose (recommended dose 370-740 MBq) from the cell suspension. The ${ }^{99 \mathrm{~m}} \mathrm{Tc}-\mathrm{HMPAO}-$ labelled WBC should be visually inspected and reinjected into the patient as soon as possible, and not later than $1 \mathrm{~h}$ after completion of the labelling procedure. Injection of the labelled WBC should be performed slowly, preferably using a needle of at least $22 \mathrm{G}(0.7 \mathrm{~mm}$ diameter $)$ to prevent cell damage due to shear stress (the inner diameter of the needle is only approximately 50-fold larger than the diameter of the WBC). Check the patient's identity prior to administration of the labelled WBC.

\section{Quality controls}

Several methods for quality control have been described, although only a few of them are used regularly in clinical routine, as many of these tests are time-consuming. For routine clinical use, visual inspection of the final product and determination of the LE are usually considered sufficient. Microscopic inspection of clumping, the trypan blue exclusion viability test and the post-release sterility test could be used as additional quality controls, when desired. These tests should be included when setting the methodology for its validation and when a new variation in the method is introduced. Early in vivo lung uptake and liver-to-spleen activity ratio are the most commonly used in vivo indices of quality control. For process validation, operator validation or periodic process control, additional functional tests such as chemotaxis or phagocytosis assays may be included, but these are not recommended for routine use.

\section{C1. Visual inspection (recommended routinely) Visual} inspection of the preparation searching for clumps, clots, fibrin and platelet aggregates should be performed throughout the whole procedure and in particular after resuspending the pellet of cells after centrifugation. At the end of the procedure and before collecting the labelled cells in the syringe for administration to the patient, the inspection should be performed carefully by gently rotating the vial. In case of aggregates, they should be dissolved by gently shaking or pipetting the sample. If clumps cannot be dissolved, the preparation should not be released for injection.

C2. Labelling efficiency (recommended routinely) After each production, the LE should be determined by measuring the amount of radioactivity in the supernatant (soluble ${ }^{99 \mathrm{~m}}$ Tc-compounds) and the pellet (cell-associated

${ }^{99 \mathrm{~m}} \mathrm{Tc}$ ) of the labelling solution after centrifugation. The LE can be calculated using the formula: $L E(\%)=$ $\frac{\text { radioactivity in pellet }}{\text { radioactivity in pellet }+ \text { radioactivity in supernatant }} \times 100 \mathrm{~A}$ LE between $40 \%$ and $80 \%$ is expected. If the LE is $<40 \%$ further quality controls should be performed, such as microscopic inspection and trypan blue exclusion test for cell viability (see step C4).

C3. Sterility (recommended periodically) For post-release sterility testing, tests should be performed in accordance with the method described in the most recent European Pharmacopoeia. This test is preferably performed by a microbiologist and may vary from centre to centre according to local needs and experience. The sterility test should be performed in triplicate for the validation of the procedure and in the case of any modification to the technique, including new personnel and new reagents. The recent availability of media fills allows validation and re-validation of the labelling procedure with sterility control of each single step of the labelling procedure. If sterility tests are not passed, the process should be revalidated. A test for contamination of solutions and reagents with pyrogens (e.g. Limulus test, LAL test, PBI International, Milan, Italy, or Endosafe-PTS, Charles River Laboratories, L'Arbresle, France) can also be applied in addition to sterility tests. The method is described in detail in the current European Pharmacopoeia.

C4. Trypan blue exclusion test, clumping and cell counting (recommended periodically) Add $25 \mu \mathrm{l}$ of $0.4 \%$ trypan blue solution in water to $25 \mu \mathrm{l}$ of the labelled leucocyte cell suspension (from step B) and gently mix the solution. Put a drop of the blue mixture into a haemocytometer and place the haemocytometer under a phase-contrast microscope at 100-fold magnification. Check the counting chamber for clumps and microaggregates of cells, count the number of cells and count the percentage of blue-stained cells (cells that have been damaged during the labelling process). As a control, repeat the same procedure using unlabelled leucocytes (from step A3). A preparation with $>4 \%$ of dead cells (blue-stained cells) should not be released for injection into the patient and new tests for validation of the method should be undertaken.

C5. Cell subset recovery test (recommended for initial validation) The test consists of counting the number of different cell subsets present during the separation and labelling procedure to verify that red blood cell and platelet contaminations are within an acceptable range. After each crucial step (i.e. after the centrifugation steps and at the end of procedure) collect a drop of resuspended cells and dispense it into a $5-\mathrm{ml}$ vial with $1 \mathrm{ml}$ saline or PBS. Count the cells using a routine cytofluorimeter for haematology or a haemocytometer slide and view under an optical microscope. Limits of acceptability in the final cell 
suspension are erythrocyte/WBC ratio $<3$ and platelet/WBC ratio $<1$.

C6. Measurement of cell efflux of ${ }^{99 m} T c$ (recommended for initial validation) After labelling, HMPAO is converted inside the cells mainly to a hydrophilic complex by reducing agents such as glutathione, thus preventing its free passage through the cell membrane. However, with time this conversion is reversible and some ${ }^{99 \mathrm{~m}} \mathrm{Tc}-\mathrm{HMPAO}$, as well as some free ${ }^{99 \mathrm{~m}} \mathrm{Tc}$, can be released by leucocytes. Damaged leucocytes may release more radioactivity and more quickly than intact cells. Efflux of ${ }^{99 m} \mathrm{Tc}$ can measured by preparing three aliquots of labelled leucocytes and incubating them at $37^{\circ} \mathrm{C}$. After $1 \mathrm{~h}$ and optionally after $4 \mathrm{~h}$ and $24 \mathrm{~h}$, an aliquot is centrifuged at $150 \mathrm{~g}$ for $10 \mathrm{~min}$ and the radioactivity in the pellet and supernatant is counted separately. A release of $<10 \%$ (i.e. radiochemical purity $>90 \%)$ at $1 \mathrm{~h}$ is acceptable [4].

C7. In vivo lung uptake (recommended routinely) Early, temporary lung uptake of labelled leucocytes may be normal. However, lung images acquired at $30 \mathrm{~min}$ after injection should show an almost complete clearance of lung activity. Focal spots of radioactivity in the lungs at $30 \mathrm{~min}$ or later indicate the presence of radiolabelled clumps of cells in the injection sample. Diffuse lung activity, intense at $30 \mathrm{~min}$ and persisting in late images is an indication of cell damage as a result of the labelling procedure, in particular in patients without any known lung disease.

In general, four different patterns of lung activity can be observed:

1. Rapid transit of cells in the lung with disappearance of radioactivity within $5 \mathrm{~min}$.

2. Delayed lung transit of labelled cells but with complete clearance within $30 \mathrm{~min}$.

3. Prolonged focal or diffuse retention of lung activity that disappears within $3 \mathrm{~h}$ of injection.

4. Delayed lung transit (as in 2) with increased liver activity greater than spleen activity.

Patterns 1 and 2 are normal. Patterns 3 and 4 indicate cell damage, the examination is nondiagnostic and particular care should be taken with image interpretation. (NB: It must be kept in mind that some disease processes can be associated with diffuse or focal lung activity and/or delayed washout of labelled WBC.)

Lung transit is a qualitative test. In case of doubt a quantitative test of liver-to-spleen ratio can be performed as described below (see step C8).

C8. In vivo liver-to-spleen ratio (recommended periodically) Normally, at any time point, spleen activity should be higher than liver activity. Tissue activity can be quantified by region of interest analysis and should be normalized to the area of the region of interest. A liver activity the same as or higher than spleen activity indicates cell damage, the scan may be nondiagnostic and particular care should be taken with image interpretation.

\section{Methodological issues requiring further clarification}

- The optimal formulation of HES plasma expander and the value of alternatives such as succinylated gelatin and methyl cellulose.

- The use of low-speed centrifugation instead of sedimentation to separate LRP from red blood cells.

- The added value of an optional additional centrifugation step to reduce the amount of contaminating platelets before labelling.

- The use of PBS or saline as an alternative to CFP as the medium for cell labelling and resuspension.

- The use of PRP instead of CFP, PBS or saline for centrifugation after labelling.

- The use of PBS as an alternative to CFP for reconstitution of labelled WBC.

- The need for gradient centrifugation and the availability of GMP grade gradient solutions for granulocyte purification.

- Qualification and training required for personnel who perform WBC labelling.

- Requirements for equipment and infrastructure for WBC labelling.

\section{Closed disposable sterile systems for WBC labelling}

The recent availability of a closed disposable sterile system (Leukokit; GE Healthcare, Little Chalfont, UK) for WBC separation and labelling offers an additional advantage for operator protection and for avoiding sample contamination. It is a licensed medical device distributed worldwide that may allow simplification of the required infrastructure, although to date there is no defined legislation for the use of this type of product in a different way from that of open systems. The kit includes a sterile GMP-produced vial of anticoagulant agent (ACD-A), a vial of $10 \%$ HES and a vial of PBS for cell washing and resuspension, thus avoiding possible causes of contamination of the labelled product.

\section{Procedure and personnel validation}

The WBC labelling process must be simulated for validation of the process prior to starting clinical studies in a specific laboratory. It is recommended that the requirements for the validation procedure are more strict 
than those for regular quality control. The validation procedure should include control of LE $(>50 \%)$, sterility test (negative), pyrogenicity (absent), viability of cells $(>98 \%)$, cell subset recovery test (in final cell suspension erythrocyte/WBC ratio $<3$ ) and measurement of in vitro cell efflux of ${ }^{99 \mathrm{~m}} \mathrm{Tc}$ within the first hour after labelling $(<10 \%)$. Sterility tests can be done with media fills or using different culture media. Tests may vary considerably and may include bacterial growth medium (e.g. agar) plates for environmental monitoring as well as hand-wash plates for hand-wash validation.

Procedure and personnel validation should be performed at least three times for each new operator prior to initiation of clinical studies and should be repeated at regular intervals (suggested every 6 months) and after any significant change in the method or reagents.

Acknowledgments The Taskgroup of the EANM on Infection/ Inflammation Imaging would like to thank the following colleagues: Marco Chianelli, Dante D'Oppido, Paola Erba, Elena Lazzeri, Napoleone Prandini and Anna Laura Viglietti from the AIMN study group on Inflammation/Infection, and Alain Faivre-Chauvet and Clemens De Cristoforo from the EANM Radiopharmacy Committee for their collaboration in the preparation of these guidelines, and their contribution through useful discussions and suggestions. We also thank Lori Camillo-Sforza for manuscript editing and administrative assistance and the ISORBE Presidium for useful suggestions.

Disclaimer The European Association of Nuclear Medicine (EANM) has written and approved guidelines to promote the use of nuclear medicine procedures of high quality. The guidelines should not be deemed inclusive of all proper procedures and exclusive of other procedures reasonably directed to obtaining the same results. The facilities in a specialized practice setting may be different from those in a more general setting. Resources available to care for patients, legislation and local regulations may vary greatly from one European country or one medical facility to another. For these reasons, these guidelines cannot be rigidly applied.

Open Access This article is distributed under the terms of the Creative Commons Attribution Noncommercial License which permits any noncommercial use, distribution, and reproduction in any medium, provided the original author(s) and source are credited.

\section{References}

1. Guidelines on current good radiopharmacy practice (cGRPP) in the preparation of radiopharmaceuticals. EANM Radiopharmacy committee. Eur J Nucl Med Mol Imaging 2009 (in press), https:// www.eanm.org/scientific_info/guidelines/gl_radioph_cgrpp.pdf

2. Ak I, Vardereli E, Durak B, Gülbaş Z, Basaran N, Stokkel MPM, et al. Labeling of mixed leukocytes with $99 \mathrm{mTc}-\mathrm{HMPAO}$ causes severe chromosomal aberrations in lymphocytes. J Nucl Med 2002;43:203-6.

3. Annovazzi A, Bagni B, Burroni L, D'Alessandria C, Signore A. Nuclear medicine imaging of inflammatory/infective disorders of the abdomen. Nucl Med Commun 2005;26:657-64.
4. Capriotti G, Chianelli M, Signore A. Nuclear medicine imaging of diabetic foot infection: results of meta-analysis. Nucl Med Commun 2006;27:757-64.

5. Cascini GL, De Palma D, Matteucci F, Biggi A, Rambaldi PF, Signore A, et al. Fever of unknown origin, infection of subcutaneous devices, brain abscesses and endocarditis. Nucl Med Commun 2006;27:213-22.

6. Danpure HJ, Osman S. A review of methods of separating and radiolabelling human leucocytes. Nucl Med Commun 1988;9:681-5.

7. Danpure HJ, Osman S, Carroll MJ. The development of a clinical protocol for the radiolabelling of mixed leucocytes with 99Tcmhexamethylpropyleneamine oxime. Nucl Med Commun 1988;9:46575.

8. Jacquier-Sarlins MR, Polla BS, Slosman DO. Oxido-reductive state: the major determinant for cellular retention of technetium99m-HMPAO. J Nucl Med 1996;37:1413-6.

9. Morrissey GJ, Powe JE. Routine application of fractional HMPAO stored at -70C for WBC scintigraphy. J Nucl Med 1993;34:151-5.

10. Palestro CJ, Brown ML, Forstrom LA, Greenspan BS, McAfee JG, Royal HD, Schauwecker DS, Seabold JE, Signore A. Society of Nuclear Medicine Procedure Guideline for $99 \mathrm{mTc}$-exametazime (HMPAO)-labeled leukocyte scintigraphy for suspected infection/ inflammation, version 3.0, 2004, http://interactive.snm.org/docs/ HMPAO_v3.pdf

11. Peters AM, Osman S, Henderson BL, Kelly JD, Danpure HJ, Hawker RJ, et al. Clinical experience with $99 \mathrm{mTc}-$ hexamethylpropyleneamineoxime for labelling leukocytes and imaging inflammation. Lancet 1986;8513:946-9.

12. Prandini N, Lazzeri E, Rossi B, Erba P, Parisella MG, Signore A. Nuclear medicine imaging of bone infections. Nucl Med Commun 2006;27:633-44.

13. Roca M, Martín-Comín J, Becker W, Bernardo-Filho M, Gutfilen B, Moisan A, et al. A consensus protocol for white blood cell labelling with technetium-99m hexamethylpropylene amine oxime. Eur J Nucl Med 1998;25:797-9.

14. Roddie ME, Peters AM, Danpure HJ, Osman S, Henderson BL, Lavender JP, et al. Inflammation: imaging with Tc-99m HMPAOlabeled leukocytes. Radiology 1988;166:767-72.

15. Sampson CB, Solanki C, Barber RW. 99Tcm-exametazime-labelled leucocytes: effect of volume and concentration of exametazime on labelling efficiency, and clinical protocol for high efficiency multidose radiolabelling. Nucl Med Commun 1991;12:719-23.

16. Saverymuttu SH, Peters AM, Dampure HJ, et al. Lung transit of 111Indium-labelled granulocytes. Relationship to labeling technique. Scand J Haematol 1983;30:151-60.

17. Solanki KK, Mather SJ, Al Janabi M, Britton KE. A rapid method for the preparation of 99Tcm hexametazime-labelled leucocytes. Nucl Med Commun 1988;9:753-61.

18. Suess E, Huck S, Reither H, Hörtnagl H, Angelberger $P$. Uptake mechanism of technetium-99m-d, 1-HMPAO in cell cultures of the dissociated postnatal rat cerebellum. J Nucl Med 1992;33:108-14.

19. Thierens HMA, Vral AM, Van Haelst JP, Van de Wiele C, Schelstraete KHG, de Ridder LIF. Lymphocyte labeling with technetium-99m-HMPAO: a radiotoxicity study using the micronucleus assay. J Nucl Med 1992;33:1167-74.

20. Piera C, Vázquez A, Hernández MA, Plancha MC, Ruiz A, Mateos JJ, et al. Método rápido de marcaja de leucocitos con 99mTc HM-PAO. Rev Esp Med Nucl 2002;21:343-8.

21. UK Radiopharmacy Group. Guidelines for the safe preparation of radiolabelled blood cells. http://www.ukrg.org.uk/

22. Yeo C, Saunders N, Locca D, Flett A, Preston M, Brookman P, et al. Ficoll-Paque versus Lymphoprep: a comparative study of two density gradient media for therapeutic bone marrow mononuclear cell preparations. Regen Med 2009;4:689-96. 